The switching of rotaxane-based motors

This article has been downloaded from IOPscience. Please scroll down to see the full text article.

2011 Nanotechnology 22205501

(http://iopscience.iop.org/0957-4484/22/20/205501)

View the table of contents for this issue, or go to the journal homepage for more

Download details:

IP Address: 141.211.173.82

The article was downloaded on 06/04/2012 at 16:52

Please note that terms and conditions apply. 


\title{
The switching of rotaxane-based motors
}

\author{
Seungjun Lee and Wei Lu${ }^{1}$ \\ Department of Mechanical Engineering, University of Michigan, Ann Arbor, MI 48109, USA \\ E-mail: weilu@umich.edu
}

Received 31 August 2010, in final form 23 February 2011

Published 28 March 2011

Online at stacks.iop.org/Nano/22/205501

\begin{abstract}
Linear molecular motors have recently attracted considerable interest. In this paper we use molecular dynamics simulations to investigate the structural and energetic properties of neutral and oxidized [2]rotaxane motors. We first consider a neutral structure to identify the stable configuration of a bistable [2]rotaxane whose ring component is on the tetrathiafulvalene (TTF) recognition site, followed by a study of the dynamic switching process of an oxidized

[2]rotaxane. The study shows that for a neutral structure the ring component stays at the TTF station in both free and constrained situations. When the station is oxidized, the ring is pushed to the other station and the dynamic switching process finishes in about $10 \mathrm{~ns}$. By comparing the results for the cases of free and fixed ends, the simulations show that structural deformation plays an important role during the switching process and can significantly affect the displacement output of molecular motors.
\end{abstract}

\section{Introduction}

Linear molecular motors have recently attracted considerable interest in the field of nanoscience [1-6]. The basic mechanism of a class of linear molecular motor known as bistable rotaxanes is as follows: the structure is composed of a dumbbell-shaped chain and encircling ring-shaped component. The chain incorporates two recognition sites whose energy state is stable when the ring component is positioned on them. If one of the recognition sites is positively charged by an external stimulus (electrochemical [7, 8] light [9-11], or $\mathrm{pH}[12,13])$, the ring switches to the other site due to the electrostatic repulsion between the ring and the oxidized station.

While different kinds of individual molecular motors such as molecular shuttles [14], valves [13], and elevators [15] are still being synthesized for different purposes, significant interest has recently focused on how to realize bulk actuation by employing molecular motors as components of nanodevices. This bottom-up approach is expected to overcome the size limit of the conventional top-down approach. For example, the concept of employing molecular motors as logical switches between metallic electrodes has been introduced [16]. The bending of a beam coated with molecular muscles which are synthesized by combining two linear molecular motors has been demonstrated [17, 18]. A

\footnotetext{
1 Author to whom any correspondence should be addressed.
}

molecular-level active plasmonic device has been made, which can be operated by switching the extinction properties [19].

In addition to experimental investigations, theoretical studies have been performed recently to reveal the physical characteristics of molecular motors. Jang et al investigated the structural characteristics of self-assembled monolayers (SAMs) of bistable rotaxanes on Au surfaces [20]. They studied several features of the SAM such as stress, tilt angle of rotaxanes and the thickness of the SAM. It showed that these features are related to the coverage density and they suggested the optimal packing density of a SAM. Jaime and collaborators investigated the effect of water and acetonitrile solvent as well as the presence of counterions on the energetic and structural characteristics of the rotaxane that incorporates two recognition sites, benzidine and 4,4'-biphenol with molecular dynamics (MD) [21, 22]. Briquet et al studied the equilibrium position of $\alpha$-CDs, the ring component, along the azobenzene type chain at an experimental temperature of $330 \mathrm{~K}$, and calculated the energy profile with a molecular mechanics (MM) method [23].

Although recent theoretical research has shed light on various kinds of structural characteristics of molecular motors [24-26], the dynamic ring switching process has not been systematically considered. While it is generally believed that charge repulsion between the ring and tetrathiafulvalene (TTF) station drives the ring motion, it is not clear quantitatively whether the repulsion is strong enough for the motion to happen and how it happens. Through molecular 
Table 1. The force field used in the MD simulation.

\begin{tabular}{llll}
\hline & Dreiding force field & LAMMPS & Modified constants used in LAMMPS \\
\hline van der Waals & $D_{0}\left\{\left(\frac{6}{\xi-6}\right) \mathrm{e}^{\xi\left(1-\frac{r}{r_{0}}\right)}-\left(\frac{\xi}{\xi-6}\right)\left(\frac{r}{r_{0}}\right)^{-6}\right\}$ & $A \mathrm{e}^{-\frac{r}{\rho}}-C r^{-6}$ & $A=D_{0}\left(\frac{6}{\xi-6}\right) \mathrm{e}^{\xi}, \rho=\frac{r_{0}}{\xi} C=D_{0}\left(\frac{\xi}{\xi-6}\right) r_{0}^{6}$ \\
Electrostatic interaction & $C \frac{q_{i} q_{j}}{\varepsilon r}$ & $C \frac{q_{i} q_{j}}{\varepsilon r}$ & Same \\
Bond stretching & $\frac{1}{2} K_{R}\left(r-r_{0}\right)^{2}$ & $K\left(r-r_{0}\right)^{2}$ & $K=\frac{1}{2} K_{R}$ \\
Angle bending & $\frac{1}{2} \frac{K_{\theta}}{\sin ^{2} \theta_{0}}\left(\cos \theta-\cos \theta_{0}\right)^{2}$ & $K\left(\cos \theta-\cos \theta_{0}\right)^{2}$ & $K=\frac{1}{2} \frac{K_{\theta}}{\sin ^{2} \theta_{0}}$ \\
Torsion & $1-\cos \left(n\left(\phi-\phi_{0}\right)\right)$ & $K(1+d \cos (n \phi))$ & Same as [18] \\
\hline
\end{tabular}

simulations, in this paper we have identified the detailed structural configuration in the switching process. In particular, we show that morphological deformation plays a key role for the successful switching of the ring. The results suggest that a fully stretched molecular motor, which is constrained from structural deformation, may not operate properly in applications. In addition, the simulations provide quantitative information such as the switching time, displacement and time evolution of energy which are hard to measure in experiments. These quantitative results will be valuable for the design and application of molecular motors. In this paper we present the energetic and structural characteristics of the dynamic switching process of oxidized [2] rotaxane using MD simulations. The atomic partial charges of the oxidized station are obtained by quantum mechanics calculations. Free and constrained situations for both neutral and oxidized structures are investigated. As a model system we consider bistable [2]rotaxanes that have two recognition sites, TTF and hydroquinone (HQ), which are $\pi$-electron rich components, and the ring, cyclobis (paraquat- $p$-phenylene) $\left(\mathrm{CBPQT}^{4+}\right)$, which is a $\pi$-electron deficient component (figure 1(a)). Figure 1(b) shows the model used in our simulations, which is built with Gaussview.

\section{Molecular dynamics simulation}

MD calculations in this work were performed with LAMMPS [27] developed at Sandia National Lab. A time step of 1 fs was used for all simulations. Cutoff distances of $8 \AA$ and $50 \AA$ were used for pair potential energy and electrostatic energy, respectively. Because the total length of the structure is about $47 \AA$, electrostatic interactions were considered directly by setting a cutoff range of $50 \AA$. The Verlet algorithm [28] was used to integrate the equations of motion. The canonical ensemble was used to control the temperature set at $300 \mathrm{~K}$ with a Nose-Hoover thermostat $[29,30]$ (relaxation time of $0.1 \mathrm{ps}$ ). The initial velocity was randomly generated in accordance with the system temperature of $300 \mathrm{~K}$.

To describe all the interactions between atoms, the Dreiding force field [31], which has been well tested in computational modeling of bistable [2] rotaxane [24, 25, 20], was employed in this simulation. The total potential energy is given by

$$
E_{\text {total }}=E_{\mathrm{vdw}}+E_{Q}+E_{\text {bond }}+E_{\text {angle }}+E_{\text {torsion }}
$$

where $E_{\mathrm{vdw}}, E_{Q}, E_{\mathrm{bond}}, E_{\mathrm{angle}}, E_{\text {torsion }}$ are the total, the van der Waals, electrostatic, bond stretching, angle bending

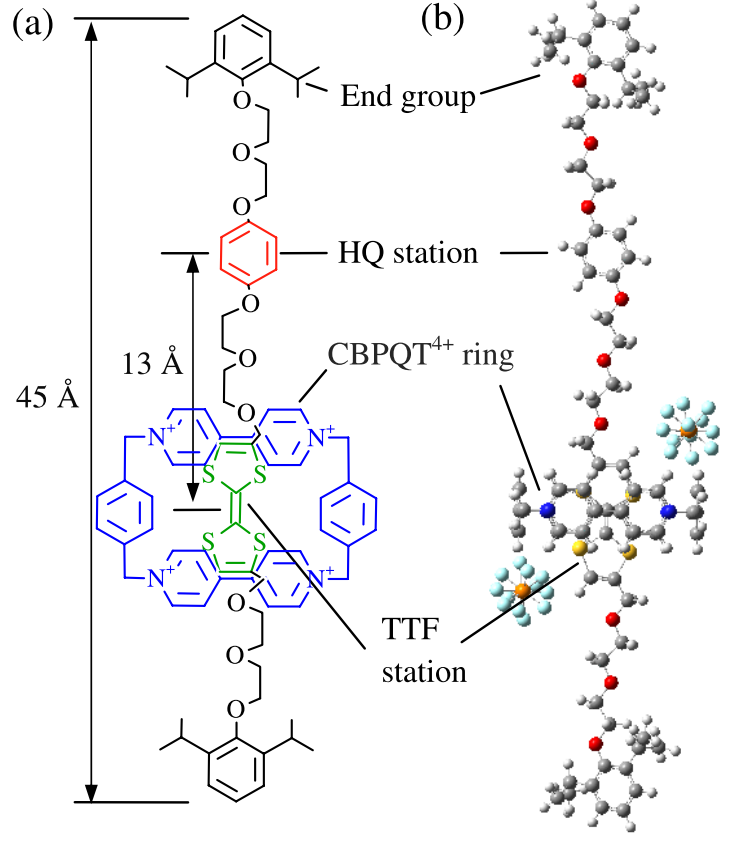

Figure 1. Bistable rotaxane studied in this paper: (a) a schematic atomic structure (TTF, green; HQ, red, CBPQT ${ }^{4+}$, blue) and (b) a computational model made with Gaussview.

and torsion energies, respectively. Although the force fields provided in LAMMPS are not identical to the Dreiding force field, simple modifications allows the use of the Dreiding force field in LAMMPS. The simulations have used the Buckingham potential for van der Waals pair potential, a harmonic bond style for bond stretching, a cosine/squared angle style for angle bending, and a harmonic dihedral style for torsion. These equations are summarized in table 1. Parameters and methods for heterogeneous atomic pair interactions follow those in [31].

To calculate the electrostatic energy, the atomic partial charges were obtained from quantum mechanical (QM) calculations using Gaussian software [32]. We used B3LYP of density functional theory with a $6-31 \mathrm{G}$ basis set. The partial charges of the ring and dumbbell structure were calculated separately. In the QM calculation the total charge of the ring was set to +4 electron charges and that of the dumbbell was set to neutral. After the partial charges were calculated, the results were fitted to each atom in the input file of LAMMPS. To simulate the dynamics of the ring movement we also calculated the atomic partial charges when the TTF station was positively 


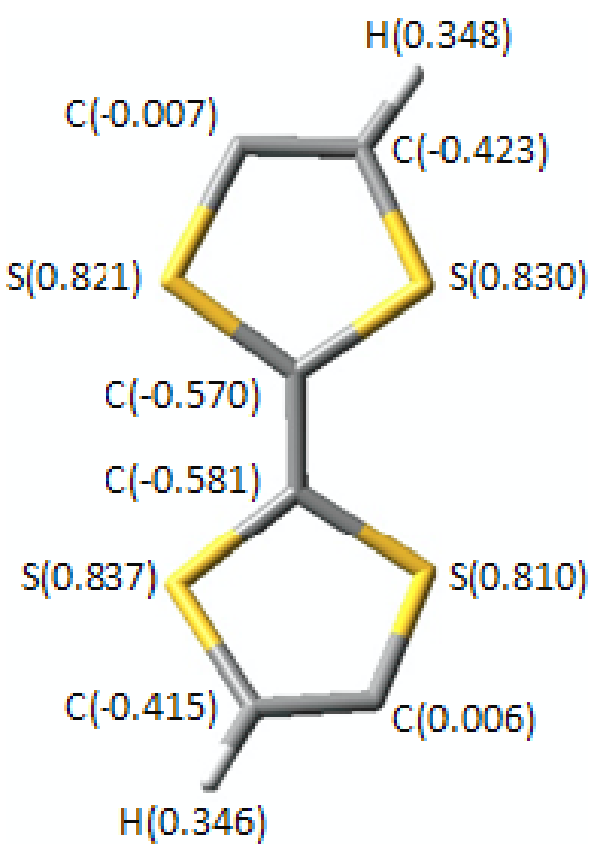

Figure 2. Atomic partial charges of the $+2 \mathrm{e}$ charged TTF station. They are calculated by Gaussian.

charged. The calculated partial charges of the TTF station are shown in figure 2 .

\section{Results and discussions}

We first performed the MD simulation for the neutral structure to determine whether the Dreiding force field can well describe the characteristics of bistable [2]rotaxane. The simulation should capture the equilibrium state when the ring is positioned at the TTF station, which is known to be the most stable state. For all simulations the ring was initially positioned at the TTF station. The partial atomic charges of the station were obtained from QM calculations by setting the entire dumbbell structure as neutral. The snapshots of the MD simulation of a free non-constrained motor are shown in figure 3. From the initial configuration to 1000 fs the ring changes from the vertical position against the dumbbell to the $45^{\circ}$ angle. Then the ends of the structure start to fold and head toward opposite directions: the left end heads upward and the right end heads downward in the figure. At time 2500 fs the structure shows a curved morphology. The ends of the structure tend to line up, which causes the structure to twist as shown for time $5000 \mathrm{fs}$. Eventually the structure appears folded. During the simulation the ring stays within the TTF station and does not move out of the station. This result confirms that a ring positioned at the TTF station is stable. The simulations suggest that a free motor may fold spontaneously so that the ring movement is not linear. For practical applications, the chain should be bonded to a substrate or other load-bearing structures, and subjected to tension. The constraint can suppress the tendency of chain folding, and provide a smooth path for the ring movement.

The change of energy is shown at the bottom of figure 3 as the simulation proceeds. The black, red and

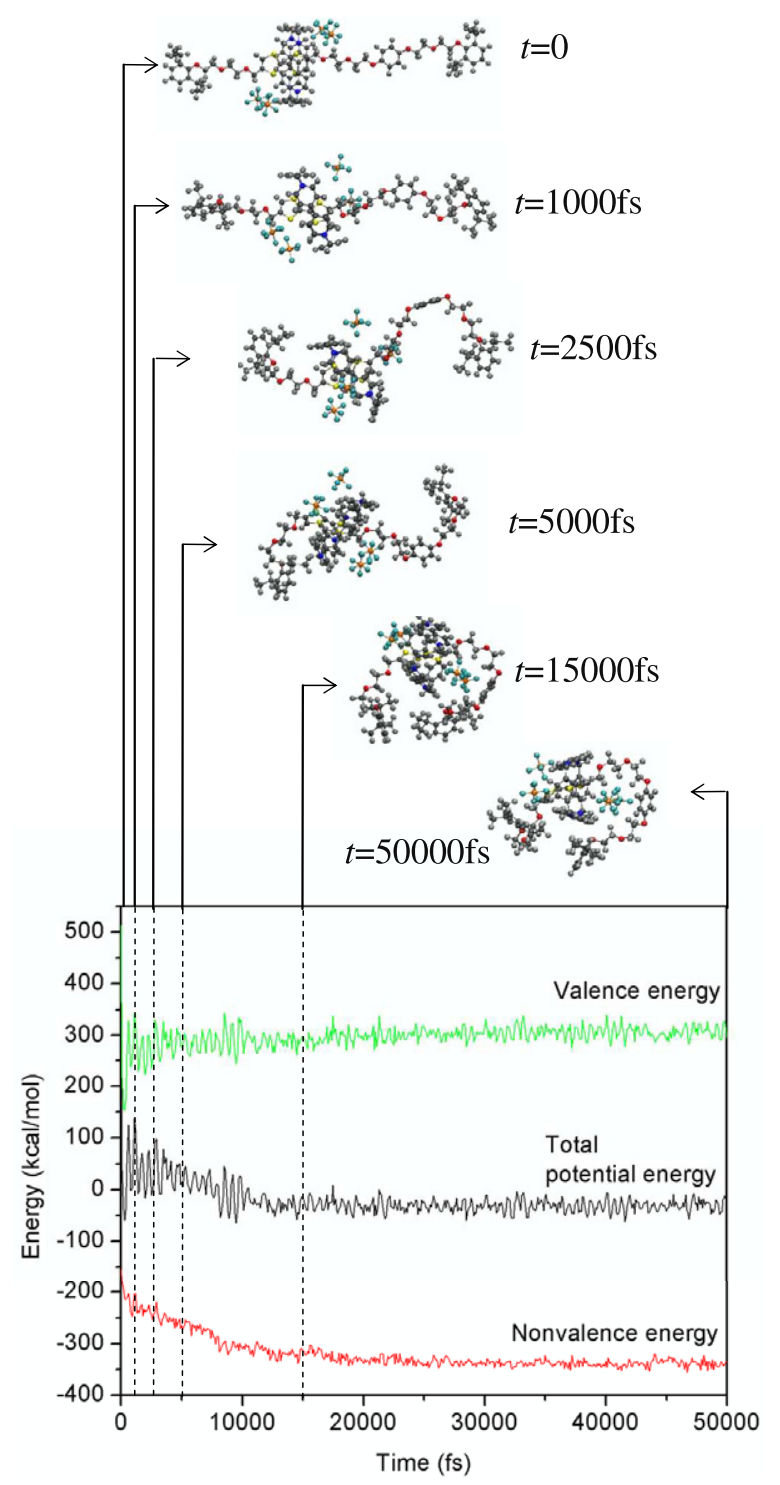

Figure 3. Conformational evolution of a neutral and unconstrained rotaxane and the time evolution of energy.

green lines represent the total potential energy, nonvalence energy (van der Waals energy + electrostatic energy) and valence energy (bond stretching + angle bending + torsion), respectively. After about $15000 \mathrm{fs}$, the total potential energy converges, which means the system is in equilibrium. The structure at time $50000 \mathrm{fs}$ has not changed much, compared with $15000 \mathrm{fs}$. The curve of the nonvalence energy follows that of the total potential energy, suggesting that it plays a key role in the equilibrium process. The large decrease in the nonvalence energy is related to folding of the structure.

Now consider the case where the two ends of a bistable motor are fixed, for example attached to a substrate. This was achieved by constraining the positions of hydrogen atoms of the two stoppers. In the simulation we set zero velocity to these atoms so that their positions did not change. Figure 4 shows the result. The ring adjusts its position and angle until it reaches an angle of about $45^{\circ}$. Similar to the unconstrained situation, the TTF station is the stable location for the ring position. 


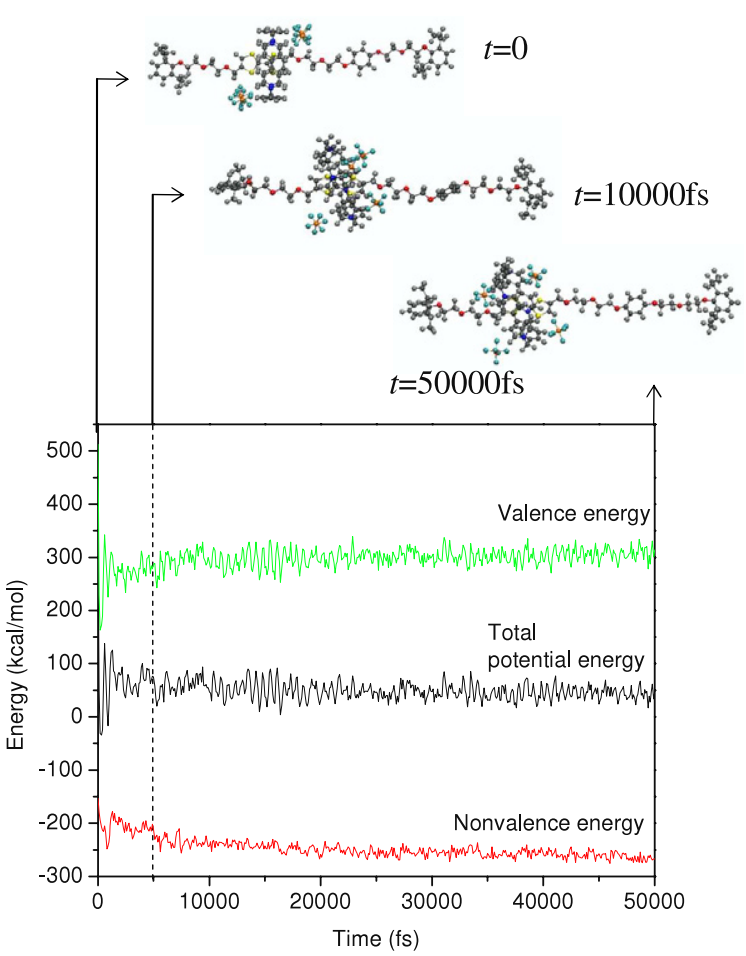

Figure 4. Conformational evolution of a neutral and constrained rotaxane and the time evolution of energy.

Compared to figure 3 , figure 4 shows a smaller decrease of the nonvalence energy due to the non-folding situation. These results show that the ring is stable at the TTF station regardless of the structure folding situation or whether the structure is attached to a substrate.

We then investigated the dynamic switching process. When one TTF station is oxidized, it carries positive charges. The electrostatic repulsion between the ring and the TTF station drives the ring to move to the other station. First, we considered the case where the two ends are not fixed. Figure 5 shows representative results. The ring was originally positioned at the TTF station. We calculated the energy change associated with TTF oxidation to be $127 \mathrm{kcal} \mathrm{mol}^{-1}$. This is the energy difference before and after the oxidation at the initial ring position. We first calculated the potential energy at the stable ring position before oxidation. Then we used quantum mechanics to get the charge distribution after oxidation, and calculated the potential energy at the same ring position. The energy difference between the two situations reflects how much additional energy is added to the system after the oxidation happens. Recent experiments have shown that the oxidation energy of similar systems but in a liquid is about $65 \mathrm{kcal} \mathrm{mol}^{-1}$ [33]. The higher value in our calculation can be attributed to the difference in the molecules studied, and the shielding effect of the liquid which reduces the measured interactive energy compared to our calculations in a vacuum. Under electrostatic repulsion, the ring leaves its original location and moves rapidly along the chain. At time $1500 \mathrm{fs}$ the ring passes through the middle of the structure. The backbone structure is slightly curved, although the deformation is not as large as shown in the neutral case. The ring arrives

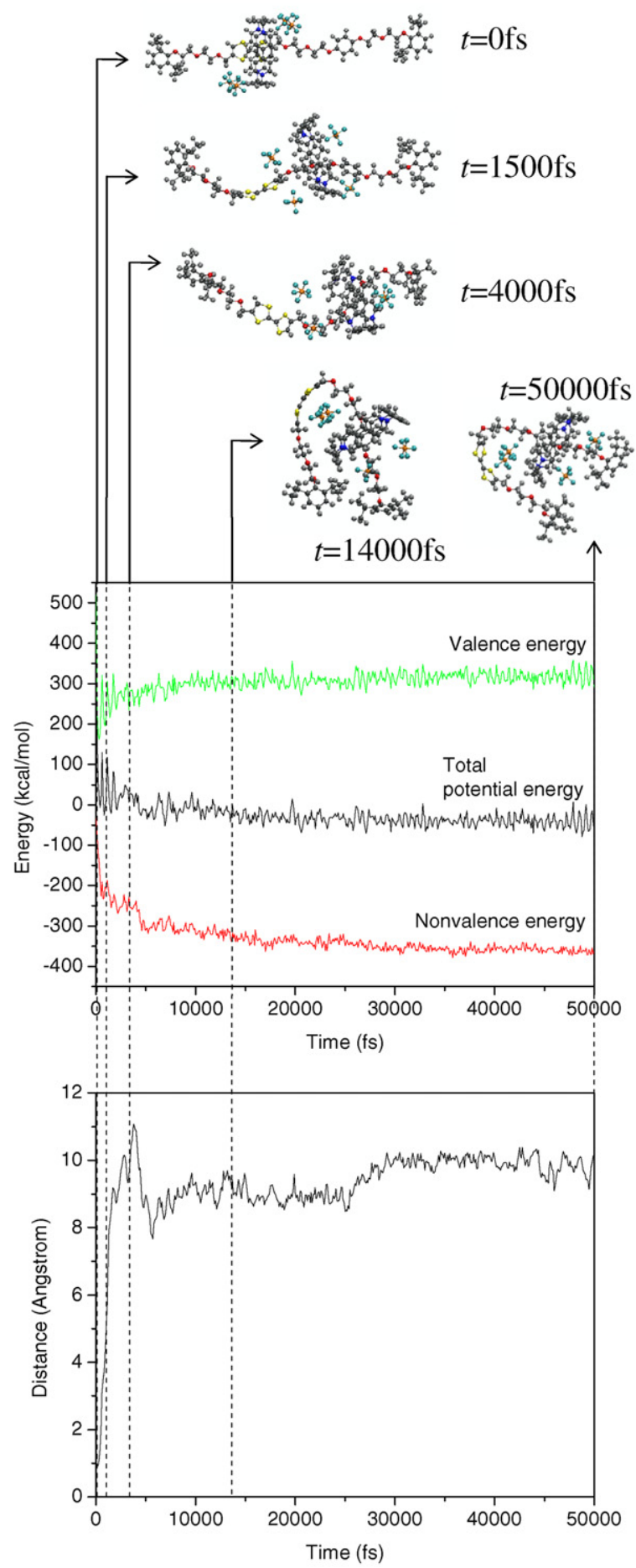

Figure 5. Conformational evolution of an oxidized and unconstrained rotaxane, as well as the time evolution of energy and the distance between the center of the ring and its original location.

at the HQ station at about 4000 fs. After it arrives at the HQ station, the ring settles down and does not seem to move further out of the station. As the ring is positioned at the HQ station, the structure starts to fold and it is totally folded at $14000 \mathrm{fs}$. The bottom of figure 5 shows the distance between the center of the ring and the TTF station as a function of time. We can see the initial overshooting and subsequent equilibration process 


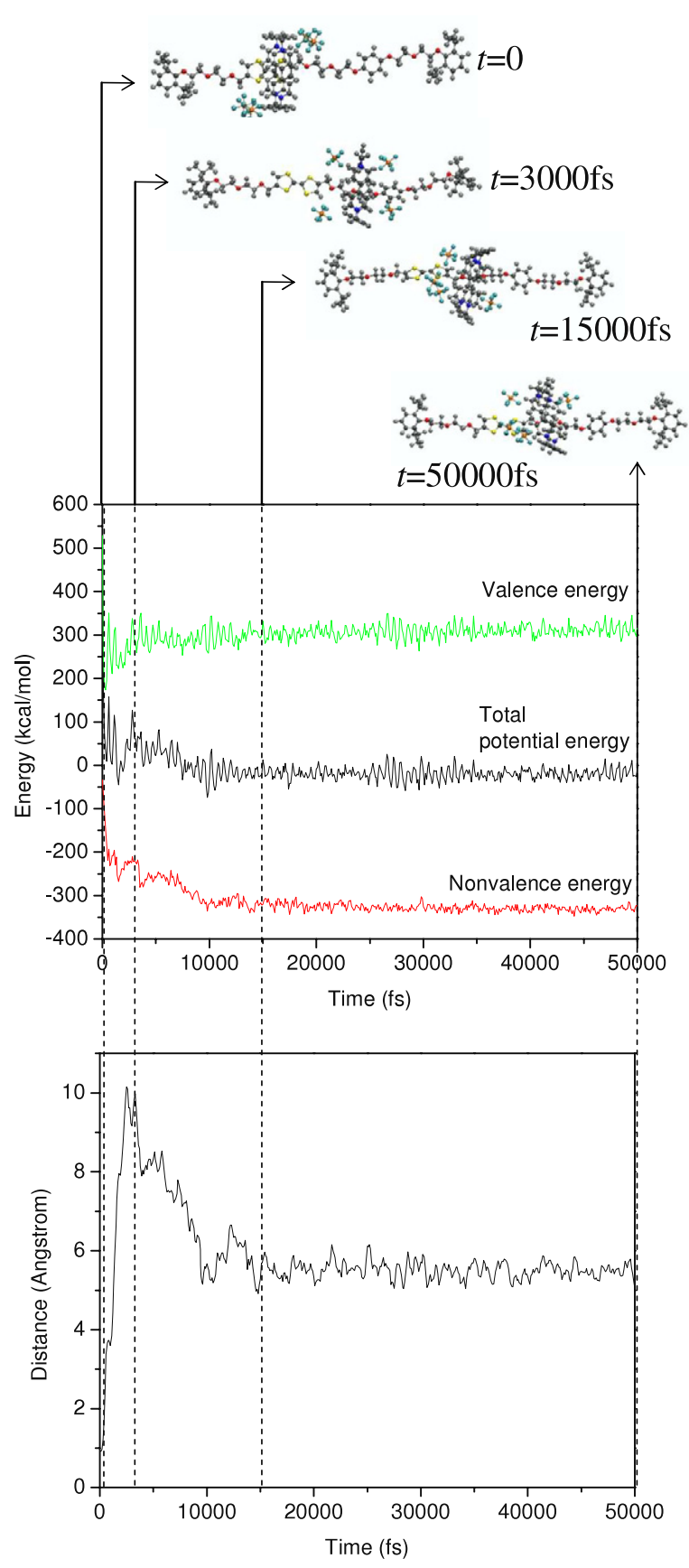

Figure 6. Conformational evolution of an oxidized and constrained rotaxane, as well as the time evolution of energy and the distance between the center of the ring and its original location.

of the ring The stable ring position is about $9 \AA$ from the TTF position. Our calculation shows that the switching happens in about 10 ps at a speed of $9 \AA / 10 \mathrm{ps}=90 \mathrm{~m} \mathrm{~s}^{-1}$, suggesting its capability for fast response applications. The slight increase in the distance after $30000 \mathrm{fs}$ is caused by the change of the ring angle.

Next we studied the case where the two ends are fixed. Figure 6 shows representative results. Because of the two fixed ends, the chain structure does not show much deformation. The ring shows tilting and morphological changes when moving to the HQ station, but maintains an integrated structure. Before reaching the HQ station, the ring moves back and then quickly settles down in the middle of the structure. No significant change in the location of the ring is observed between $15000 \mathrm{fs}$ and $100000 \mathrm{fs}$. At the bottom of figure 5, the distance between the center of the ring and the TTF station is shown as a function of time. We can see that the ring overshoot is larger than for the case of free ends. The stable ring position is about $5.5 \AA$ from the TTF position.

Our simulations have revealed interesting features during the switching process. The ring achieves a displacement of $9 \AA$ when the ends are free. This is less than the original distance between the two stations, which is about $13 \AA$. The difference is due to the folding of the structure while the ring actually moves from the TTF station to the HQ station. We expected that a larger displacement might be obtained if the ends are fixed to prevent folding. However, the displacement is shorter compared to the result for free ends. The ring does not arrive at the HQ station but settles down in the middle of the structure. This result suggests that structural deformation plays an important role in the switching process. If the structure is stretched and fixed to prevent any deformation, the ring cannot overcome the energy barrier between the two stations and will get stuck during the process. In many applications molecular motors are usually attached to a solid surface. It should be cautioned that a fully stretched motor may not function as desired. For optimal operation, a certain flexibility of the backbone should be allowed when the ends of the motors are bonded to the surface.

In summary, we have performed a structural and energetic analysis of a bistable molecular motor when the TTF station is neutral or charged, and of the dynamic ring switching process when the station is oxidized by MD simulations. For the neutral structure the ring component stays at the TTF station in both free and constrained situations. The dynamic switching process happens in about $10 \mathrm{~ns}$. The simulation also shows the importance of structural deformation during the switching process. Bistable [2] rotaxanes may be assembled into active materials for various actuator applications. They may also connect in series, the ring of one rotaxane being attached to the end group of the other, to form a daisy-chain molecular muscle, which can generate a large displacement upon actuation.

\section{Acknowledgment}

The authors acknowledge financial support from National Science Foundation Award no. CMMI-0700048.

\section{References}

[1] Balzani V, Credi A, Raymo F M and Stoddart J F 2000 Artificial molecular machines Angew. Chem. 39 3348-91

[2] Balzani V, Gomez-Lopez M and Stoddart J F 1998 Molecular machines Acc. Chem. Res. 31 405-14

[3] Ballardini R, Balzani V, Credi A, Gandolfi M T and Venturi M 2001 Artificial molecular-level machines: which energy to make them work? Acc. Chem. Res. 34 445-55

[4] Klajn R, Stoddart J F and Grzybowski B A 2010 Nanoparticles functionalised with reversible molecular and supramolecular switches Chem. Soc. Rev. 39 2203-37 
[5] Angelos S, Johansson E, Stoddart J F and Zink J I 2007 Mesostructured silica supports for functional materials and molecular machines Adv. Funct. Mater. 17 2261-71

[6] Huang T J 2008 Recent developments in artificial molecular machine-based active nanomaterials and nanosystems MRS Bull. 33226

[7] Juluri B K, Kumar A S, Liu Y, Ye T, Yang Y-W, Flood A H, Fang L, Stoddart J F, Weiss P S and Huang T J 2009 A mechanical actuator driven electrochemically by artificial molecular muscles ACS Nano 3 291-300

[8] Ye T, Kumar A S, Saha S, Takami T, Huang T J, Stoddart J F and Weiss P S 2010 Changing stations in single bistable rotaxane molecules under electrochemical control ACS Nano 4 3697-701

[9] Brouwer A M, Frochot C, Gatti F G, Leigh D A, Mottier L C, Paolucci F, Roffia S and Wurpel G W H 2001 Photoinduction of fast, reversible translational motion in a hydrogen-bonded molecular shuttle Science $2912124-8$

[10] Serreli V, Lee C-F, Kay E R and Leigh D A 2007 A molecular information ratchet Nature 445 523-7

[11] Berna J, Leigh D A, Lubomska M, Mendoza S M, Perez E M, Rudolf P, Teobaldi G and Zerbetto F 2005 Macroscopic transport by synthetic molecular machines Nat. Mater. $4704-10$

[12] Anelli P L, Spencer N and Stoddart J F 1991 A molecular shuttle J. Am. Chem. Soc. 113 5131-3

[13] Nguyen T D, Leung K C F, Liong M, Pentecost C D, Stoddart J F and Zink J I 2006 Construction of a pH-driven supramolecular nanovalve Org. Lett. $83363-6$

[14] Yoon I et al 2009 Functionally rigid and degenerate molecular shuttles Chem.-Eur. J. 15 1115-22

[15] Badjic J D, Ronconi C M, Stoddart J F, Balzani V, Silvi S and Credi A 2006 Operating molecular elevators J. Am. Chem. Soc. 128 1489-99

[16] Collier C P, Wong E W, Belohradsk, yacute M, Raymo F M, Stoddart J F, Kuekes P J, Williams R S and Heath J R 1999 Electronically configurable molecular-based logic gates Science 285 391-4

[17] Tony Jun H, Branden B, Chih-Ming H, Yi L, Amar H F, Paul A B, Hsian-Rong T, Stoddart J F, Marko B and Sergei M 2004 A nanomechanical device based on linear molecular motors Appl. Phys. Lett. 85 5391-3

[18] Liu Y et al 2005 Linear artificial molecular muscles J. Am. Chem. Soc. 127 9745-59

[19] Zheng Y B, Yang Y-W, Jensen L, Fang L, Juluri B K, Flood A H, Weiss P S, Stoddart J F and Huang T J 2009
Active molecular plasmonics: controlling plasmon resonances with molecular switches Nano Lett. 9 819-25

[20] Jang S S et al 2005 Structures and properties of self-assembled monolayers of bistable [2] rotaxanes on $\mathrm{Au}(111)$ surfaces from molecular dynamics simulations validated with experiment J. Am. Chem. Soc. 127 1563-75

[21] Grabuleda X, Ivanov P and Jaime C 2003 Computational studies on pseudorotaxanes by molecular dynamics and free energy perturbation simulations J. Org. Chem. 68 1539-47

[22] Grabuleda X, Ivanov P and Jaime C 2003 Shuttling process in [2]rotaxanes. Modeling by molecular dynamics and free energy perturbation simulations J. Phys. Chem. B $1077582-8$

[23] Briquet L, Staelens N, Leherte L and Vercauteren D P 2007 Structural, energetic, and dynamical properties of rotaxanes constituted of [alpha]-cyclodextrins and an azobenzene chain J. Mol. Graph. Model 26104

[24] Jang Y H, Jang S S and Goddard W A 2005 Molecular dynamics simulation study on a monolayer of half [2]rotaxane self-assembled on $\mathrm{Au}(111) \mathrm{J}$. Am. Chem. Soc. 127 4959-64

[25] Jang S S et al 2005 Molecular dynamics simulation of amphiphilic bistable [2] rotaxane langmuir monolayers at the air/water interface J. Am. Chem. Soc. 127 14804-16

[26] Jang S S, Lin S-T, Maiti P K, Blanco M, Goddard W A, Shuler P and Tang Y 2004 Molecular dynamics study of a surfactant-mediated decane-water interface: \& nbsp; effect of molecular architecture of alkyl benzene sulfonate J. Phys. Chem. B 108 12130-40

[27] Plimpton S 1995 Fast parallel algorithms for short-range molecular dynamics J. Comput. Phys. 1171

[28] Verlet L 1967 Computer 'experiments' on classical fluids. I. Thermodynamical properties of Lennard-Jones molecules Phys. Rev. 15998

[29] Shuichi N 1984 A unified formulation of the constant temperature molecular dynamics methods J. Chem. Phys. 81 511-9

[30] Hoover W G 1985 Canonical dynamics: equilibrium phase-space distributions Phys. Rev. A 311695

[31] Mayo S L, Olafson B D and Goddard W A III 1990 DREIDING: a generic force field for molecular simulations J. Phys. Chem. 94 8897-909

[32] www.gaussian.com

[33] Brough B, Northrop B H, Schmidt J J, Tseng H R, Houk K N, Stoddart J F and Ho C M 2006 Evaluation of synthetic linear motor-molecule actuation energetics Proc. Natl Acad. Sci. USA 103 8583-8 